\title{
On the Probability of the Number of Solutions for the P4P Problem*
}

\author{
Xiao-Shan Gao \\ Key Laboratory of Mathematics Mechanization \\ Institute of Systems Science \\ Academia Sinica, Beijing 100080, China \\ Jianliang Tang \\ College of Science, Shenzhen University, Shenzhen 518060, China
}

\begin{abstract}
This paper studies the multi-solution phenomenon for the perspective four point $(\mathrm{P} 4 \mathrm{P})$ problem from geometric and algebraic aspects. We give a pure geometric proof that the $\mathrm{P} 4 \mathrm{P}$ problem could have up to five solutions. We also give a clear picture on how these five solutions could be realized. We prove that with probability one, the $\mathrm{P} 4 \mathrm{P}$ problem has a unique solution which can be represented by a set of rational functions in the parameters. The simulant experiments show that to solve the $\mathrm{P} 4 \mathrm{P}$ problem with the rational functions is stable and accurate.
\end{abstract}

Keywords: Camera calibration, pose determination, perspective four point problem, P4P, probability, number of solutions.

\section{Introduction}

One of the fundamental goals of computer vision is to discover properties that are intrinsic to a scene by one or several images of this scene[3, 7,14$]$. Within this paradigm, an essential process is the determination of the position and orientation of the sensing device (the camera) with respect to objects in the scene [2]. This problem is known as the perspective $n$ point $(\mathrm{P} n \mathrm{P})$ problem and has many applications in automation, image

*Partially supported by a National Key Basic Research Project of China and by a USA NSF grant CCR-0201253. 
analysis, automated cartography, photogrammetry, robotics and model based machine vision system. Fishler and Bolles [3] summarized the problem as follows:

"Given the relative spatial locations of $n$ control points, and given the angle to every pair of control points from an additional point called the Center of Perspective $\left(C_{P}\right)$, find the lengths of the line segments joining $C_{P}$ to each of the control points."

One of the major concerns of the $\mathrm{P} n \mathrm{P}$ problem is its multi-solution phenomenon. The reason is that if the solution is not unique then we need further determine which solution is the one we want. Unfortunately, all $\mathrm{P} n \mathrm{P}$ problems for $n \leq 5$ have multiple solutions. It is well-known that the P3P problem could have one to four essentially different solutions $[1,3,5,12,21,20,18]$.

In order to obtain a unique solution, one natural way is to add one more control point to consider a $\mathrm{P} 4 \mathrm{P}$ problem. In $[15,16]$, the $\mathrm{P} 4 \mathrm{P}$ problem was reduced to a system of polynomial equations and then a set of solutions was found under the assumption that there exists one solution for the $\mathrm{P} 4 \mathrm{P}$ problem in normal circumstances. Algorithms for solving the $\mathrm{P} 4 \mathrm{P}$ problem were proposed in $[9,17]$ without considering the number of solutions. When the control points are coplanar, the $\mathrm{P} 4 \mathrm{P}$ problem has a unique solution [1]. If the control points are not coplanar, the $\mathrm{P} 4 \mathrm{P}$ problem could have up to five solutions [10]. It is proved that the P5P problem could have two solutions [11]. For $n \geq 6$, the $\mathrm{P} n \mathrm{P}$ problem has one solution and can be solved with the DLT method [4].

For the $\mathrm{P} 4 \mathrm{P}$ problem, we first give a pure geometric proof that the $\mathrm{P} 4 \mathrm{P}$ problem has up to five solutions. This proof gives a clear picture on how the five solutions could be achieved. Previous work makes the assumption that in the general case the P4P problem has a unique solution $[10,15,16]$. In [10], this fact is also supported by the computation of a large set of data. In this paper, we give a proof that the probability for the $\mathrm{P} 4 \mathrm{P}$ problem to have a unique solution is one. This result provides a theoretical support for the algorithms for solving $\mathrm{P} 4 \mathrm{P}$ problems, which are based on the assumption that the $\mathrm{P} 4 \mathrm{P}$ problem generally has one solution. To show this property, it is not enough to say that adding more constraints to the P3P problem will lead to constraints among the parameters. Geometrically, we need to prove the following fact. Let $N$ be the set of parameters where the $\mathrm{P} 4 \mathrm{P}$ problem has solutions, and $M$ the set of parameters where the $\mathrm{P} 4 \mathrm{P}$ problem has more than one solutions. Then $M$ must be of less dimension than that of $N$. In certain sense, this is to say that $M$ is a subset of $N$ with zero "volume". We further show that this unique solution could be represented by a set of rational functions in the parameters, and to solve the $\mathrm{P} 4 \mathrm{P}$ problem with the rational functions is stable and accurate. Similar results are also true for the P5P problem. 
The rest of the paper is organized as follows. In Section 2, give a geometric proof that the $\mathrm{P} 4 \mathrm{P}$ problem has up to five solutions. In Section 3, we prove the main result about the P4P problem. In Section 4, conclusions are presented. The proof for the main theorem is given in the appendix.

\section{Number of solutions for P4P problem}

Lemma 2.1 If the four control points of the P4P problem are not on the same plane then it could only have a finite number of solutions.

Proof. Let the four control points be $A, B, C$ and $D$. The P3P problem with center of perspective $P$ and control points $A, B, C$ has an infinite number of solutions if and only if $P$ is on the circumscribed circle of triangle $A B C$ [5]. Thus, the $\mathrm{P} 4 \mathrm{P}$ problem has an infinite number of solutions if and only if point $P$ is on the circumscribed circles of triangles $A B C, A B D, A C D, B C D$, which could happen if and only if points $A, B, C, D$ and $P$ are on the same circle. This contradicts to the fact that $A, B, C, D$ are not co-planar. I

Lemma 2.2 Let $P$ and $Q$ be solutions of the $P 4 P$ problem with non-planar control points $A, B, C$ and $D$. If $P$ and $Q$ are symmetric with respect to plane $A B C$, then $D$ must be on line $P Q$.

Proof. Since the angles between line $P D$ and the lines $P A, P B$ and $P C$ are given, $P D$ is the intersection line of three cones with $P A, P B$ and $P C$ as the central axes respectively. $P D$ is the unique intersection line of the three cones since points $A, B, C$ are not collinear. Similarly, $Q D$ is also the unique intersection line of three cones $Q_{A}, Q_{B}, Q_{C}$ with $Q A, Q B$, $Q C$ as central axes. Suppose that $D$ is not on line $P Q$. Let line $P D$ meet plane $A B C$ at point $\bar{D}$. Since $A, B, C, D$ are not co-planar, $\bar{D} \neq D$. Since $D$ is not on the line $P Q, Q D$ and $Q \bar{D}$ are different lines. Because $P$ and $Q$ are symmetric with plane $A B C$, we have $\angle A Q \bar{D}=\angle A P \bar{D}=\angle A P D=\angle A Q D$. Similarly, $\angle B Q \bar{D}=\angle B Q D, \angle C Q \bar{D}=\angle C Q D$. In other words, $Q \bar{D}$ is also the intersection of the three cones $Q_{A}, Q_{B}, Q_{C}$. This contradicts to the uniqueness of the intersection line. Therefore, $D$ must be on line $P Q$. I

Theorem 2.3 The P4P problem with non co-planar control points $A, B, C, D$ could have up to five solutions. If it has five solutions, then there exists one pair of solutions which are symmetric with one of the planes $A B C, A B D, A C D$ or $B C S$ and among the other three solutions there exist no two which are symmetric with this plane. 
Proof. By Lemma 2.1, the P4P problem could only have a finite number of solutions. There are at most eight solutions for the P3P problem with control points $A, B$ and $C$, of which four solutions are above the plane $A B C$ and four are under the plane $A B C$ symmetrically. The solutions of the $\mathrm{P} 4 \mathrm{P}$ problem must be from these eight solutions. By Lemma 2.2, if two solutions $P$ and $Q$, symmetric with respect to plane $A B C$, are both solutions of the $\mathrm{P} 4 \mathrm{P}$ problem, then $D$ is on line $P Q$. It is clear that no other solutions can be on line $P Q$. It is impossible for another pair of solutions symmetric with plane $A B C$ of the $\mathrm{P} 3 \mathrm{P}$ problem to be the solutions of the $\mathrm{P} 4 \mathrm{P}$ problem, since point $D$ is already on line $P Q$. Then the $\mathrm{P} 4 \mathrm{P}$ problem could have at most one pair of solutions symmetric with plane $A B C$. As a consequence, the $\mathrm{P} 4 \mathrm{P}$ problem has at most five solutions. In the following table, we give a concrete $\mathrm{P} 4 \mathrm{P}$ problem which has five solutions (Fig. 1 and Fig. 2). Therefore, the $\mathrm{P} 4 \mathrm{P}$ problem could have up to five solutions.

I

\begin{tabular}{|c|c|c|c|c|c|c|}
\hline Parameter & $|A B|$ & $|A C|$ & $|B C|$ & $|A D|$ & $|C D|$ & $|B D|$ \\
\hline value & 1 & 1 & 1 & $\frac{\sqrt{13}}{6}$ & $\frac{\sqrt{13}}{6}$ & $\frac{\sqrt{13}}{6}$ \\
\hline Parameter & $\cos \angle B P C$ & $\cos \angle A P C$ & $\cos \angle A P B$ & $\cos \angle C P D$ & $\cos \angle A P D$ & $\cos \angle B P D$ \\
\hline value & $\frac{5}{8}$ & $\frac{5}{8}$ & $\frac{5}{8}$ & $\frac{\sqrt{3}}{2}$ & $\frac{\sqrt{3}}{2}$ & $\frac{\sqrt{3}}{2}$ \\
\hline
\end{tabular}

Table 1. Parametric values for which the $\mathrm{P} 4 \mathrm{P}$ problem has 5 solutions

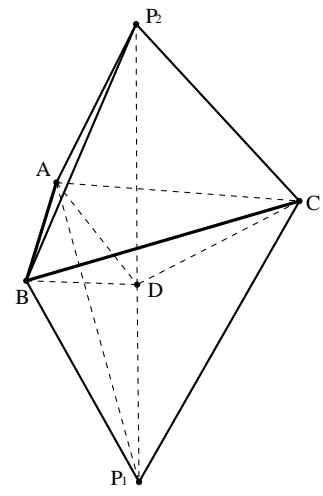

Figure 1: Two solutions symmetric with plane $A B C$

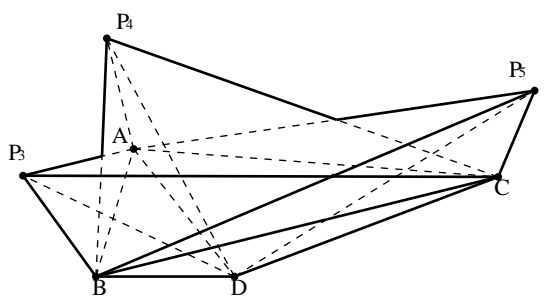

Figure 2: Three other solutions

\section{Solving the $\mathrm{P} 4 \mathrm{P}$ problem}

\subsection{Probabilities for the number of solutions}

Let $P$ be the center of perspective, and $A, B, C, D$ the control points. Let $|P A|=$ $X,|P B|=Y,|P C|=L,|P D|=Z, l_{1}=|A B|^{2}, l_{2}=|A C|^{2}, l_{3}=|B C|^{2}, l_{4}=|A D|^{2}, l_{5}=$ $|C D|^{2}, l_{6}=|B D|^{2}, p=2 \cos \angle(B P C), q=2 \cos \angle(A P C), r=2 \cos \angle(A P B)$, 
$s=2 \cos \angle(C P D), t=2 \angle(A P D), u=2 \cos \angle(B P D)$. The $P 4 P$ equation system is:

$$
\left\{\begin{array}{l}
p_{1}=X^{2}+Y^{2}-X Y r-l_{1}=0 \\
p_{2}=X^{2}+L^{2}-X L q-l_{2}=0 \\
p_{3}=Y^{2}+L^{2}-Y L p-l_{3}=0 \\
p_{4}=X^{2}+Z^{2}-X Z s-l_{4}=0 \\
p_{5}=Z^{2}+L^{2}-Z L t-l_{5}=0 \\
p_{6}=Y^{2}+Z^{2}-Y Z u-l_{6}=0
\end{array}\right.
$$

We will explain that the solutions of the $\mathrm{P} 4 \mathrm{P}$ problem for four non-coplanar points $A, B, C, D$ correspond to the positive solutions of equation system (1) for $X, Y, Z$, and $L$. It is clear that the solutions of the $\mathrm{P} 4 \mathrm{P}$ problem must be solutions of the P3P problem with points $A, B, C$. As shown by Theorem 2.3, if two of the solutions $P_{1}$ and $P_{2}$ are on different sides of plane $A B C$, then points $P_{1}, P_{2}, D$ are collinear. Since $A, B, C, D$ are not co-planar, this guarantees that $P_{1}$ and $P_{2}$ are different solutions of equation system (1). Therefore, we did not miss any solution with equation system (1).

Let $l_{1}=a w L^{2}, l_{2}=b w L^{2}, l_{3}=w L^{2}, l_{4}=c w L^{2}, l_{5}=d w L^{2}, l_{6}=e w L^{2}, X=x L$, $Y=y L, Z=z L$. Equation system (1) becomes the following equation system

$$
\left\{\begin{array}{l}
q_{1}=x^{2}+y^{2}-x y r-a w=0 \\
q_{2}=y^{2}+1-y p-b w=0 \\
q_{3}=x^{2}+1-x q-w=0 \\
q_{4}=x^{2}+z^{2}-x z s-c w=0 \\
q_{5}=z^{2}+1-z t-d w=0 \\
q_{6}=y^{2}+z^{2}-y z u-e w=0
\end{array}\right.
$$

We assume the "reality conditions" (See [5]) for the P3P problems with perspective center $P$ and control points $A, B, C ; A, B, D ; A, C, D$; and $B, C, D$ respectively. Basically speaking, we assume that each of the above triangles is proper and $P$ is not on the planes determined by these triangles.

Lemma 3.1 For a set of values of the parameters $\mathcal{V}=\left\{l_{1}, l_{2}, l_{3}, l_{4}, l_{5}, l_{6}, p, q, r, s, t, u\right\}$, the number of positive solutions of equation system (1) is the same as that of (2).

Proof. Since $|q|=|2 \cos (\angle A P C)|<2$, we have $w=x^{2}+1-x q=(x-q / 2)^{2}+1-q^{2} / 4>0$. We thus may establish the following one to one correspondence between the positive solutions of (1) and (2)

$$
(X, Y, L, Z) \stackrel{x=X / L, y=Y / L, z=Z / L, w=l_{3} / L^{2}}{\longrightarrow}(x, y, z, w) \stackrel{L=\sqrt{l_{3} / w}, X=x L, Y=y L, Z=z L}{\longrightarrow}(X, Y, L, Z) . \mathbf{I}
$$

Definition 3.2 A set of parametric values for $\mathcal{U}=\{a, b, c, d, e, p, q, r, s, t, u\}$ is called physical if equation system (2) has at least one set of positive solution. 
Let $\mathbf{P}$ be the set of physical parametric values for parameters $\mathcal{U}$. For $\mathcal{U}_{0} \in \mathbf{P}$, let $\mathbf{Z}_{\mathcal{U}_{0}}$ be the set of positive solutions of equation system (2) for parametric values $\mathcal{U}_{0}$.

Theorem 3.3 Use the notations introduced above.

1. For all $\mathcal{U}_{0} \in \mathbf{P}$, the probability for $\left|\mathbf{Z}_{U_{0}}\right|=1$ is one. In other words, for physical parameters, the probability for the P 4 P problem to have a unique solution is one.

2. We may find rational functions $P, Q, R$ in the parameters such that with a probability one for all physical parameters, the solutions for the $P 4 P$ problem can be obtained as follows $x=P, y=Q, z=R$.

To explain the above theorem, we need some concepts which may be found in $[8,19]$. Here we use examples to illustrate the concepts. For a set of polynomials $P S$ and a polynomial $D$, we use $\operatorname{Zero}(P S)$ to denote the set of real solutions for equations $P S=0$ and $\operatorname{Zero}(P S / D)=\operatorname{Zero}(P S)-\operatorname{Zero}(D) \cdot \operatorname{Zero}(P S / D)$ is called a quasi-variety. We may add inequalities to a quasi-variety to obtain semi-algebraic sets. The dimension of a quasi variety or a semi algebraic set is the number of variables that can take arbitrary values. For instance, the half unit sphere $H=\operatorname{Zero}\left(\left\{x^{2}+y^{2}+z^{2}-1\right\}\right) \cap\{(x, y, z) \mid z \geq 0\}$ is a semi-algebraic set of dimension two; the unit circle $C=\operatorname{Zero}\left(\left\{x^{2}+y^{2}-1, z\right\}\right)$ is a quasi-variety of dimension one. Since the dimension for $C$ is one, its area is zero. While the area for $H$ is $2 \pi$. Then the probability for a random point $P \in H$ belongs to $C$ is zero and the probability for a point $P \in H$ belongs to $H-C$ is one.

For the $\mathrm{P} 4 \mathrm{P}$ problem, let $\mathbf{P}$ be the set of physical parametric values for parameters $\mathcal{U}$ and $\mathbf{T}$ a set of parametric values such that the $\mathrm{P} 4 \mathrm{P}$ has one solution in $\mathbf{P}-\mathbf{T}$. We will prove that $\mathbf{P}$ is of dimension nine and $\mathbf{T}$ is of lower dimension than that of $\mathbf{P}$. Since $\mathbf{P}$ and $\mathbf{T}$ are quasi-algebraic sets, inside any sufficiently large finite box $B, B \cap \mathbf{P}$ has a positive volume as a nine-dimensional manifold and $B \cap \mathbf{T}$ has a zero volume. Since $B$ can be of any size, we may conclude that the probability for a random parametric point from $\mathbf{P}$ lies in $\mathbf{P}-\mathbf{T}$ is one. With these concepts, Theorem 3.3 is a consequence of the following result.

Theorem 3.4 The physical parameter set $\mathbf{P}$ for the P4P problem is a semi-algebraic set of dimension nine in $\mathbb{R}^{11}$. We may find a subset $\mathbf{T}$ of $\mathbf{P}$ with dimension less than nine and rational functions $P, Q, R$ in the parameters $\mathcal{U}$ such that for $\mathcal{U}_{0} \in \mathbf{P}-\mathbf{T}$, the equation system (2) has one solution which can be given by $x=P, y=Q, z=R$.

The proof can be found in the appendix. In what below, we will show how to compute the three rational functions $P, Q, R$ and give a sketch of the proof. 
For polynomials $f$ and $g$, let $r=\operatorname{Res}(f, g, x)$ be the resultant of $f$ and $g$ with variable $x$ [13]. Eliminating $w$ from (2) with $q_{3}=0$, we have the following equivalent equations:

$$
\left\{\begin{array}{l}
h_{1}=x^{2}+y^{2}-x y r-a\left(x^{2}+1-x q\right)=0 \\
h_{2}=y^{2}+1-y p-b\left(x^{2}+1-x q\right)=0 \\
h_{3}=x^{2}+z^{2}-x z s-c\left(x^{2}+1-x q\right)=0 \\
h_{4}=z^{2}+1-z t-d\left(x^{2}+1-x q\right)=0 \\
h_{5}=y^{2}+z^{2}-y z u-e\left(x^{2}+1-x q\right)=0
\end{array}\right.
$$

Let $f_{1}=h_{1}-h_{2}, f_{2}=\operatorname{Res}\left(f_{1}, h_{1}, y\right), f_{3}=h_{3}-h_{4}, f_{4}=\operatorname{Res}\left(f_{3}, h_{3}, z\right)$. Then

$$
\left\{\begin{array}{l}
f_{1}=(p-r x) y+(1-a+b) x^{2}+(q a-b q) x-a+b-1 \\
f_{2}=a_{0} x^{4}+a_{1} x^{3}+a_{2} x^{2}+a_{3} x+a_{4} \\
f_{3}=(t-s x) z+(d-c+1) x^{2}+(c q-d q) x-c+d-1 \\
f_{4}=b_{0} x^{4}+b_{1} x^{3}+b_{2} x^{2}+b_{3} x+b_{4}
\end{array}\right.
$$

where

$$
\left\{\begin{array}{l}
a_{0}=b^{2}+a^{2}-b r^{2}-2 a b+2 b+1-2 a \\
a_{1}=-2 a^{2} q+(4 b q+p r+2 q) a-p r+p r b-2 b^{2} q-2 b q+b q r^{2} \\
a_{2}=\left(2+q^{2}\right) a^{2}+\left(-p r q-p^{2}-4 b-2 q^{2} b\right) a-2+2 b^{2}-b r^{2}+b^{2} q^{2}+r^{2}-p r b q+p^{2} \\
a_{3}=-2 a^{2} q+\left(p^{2} q+p r-2 q+4 b q\right) a-p r-2 b^{2} q+2 b q+p r b \\
a_{4}=1+2 a-2 b+b^{2}+a^{2}-2 a b-p^{2} a \\
b_{0}=d^{2}+c^{2}-2 c d+2 d-d s^{2}-2 c+1 \\
b_{1}=-2 d^{2} q+\left(-2 q+q s^{2}+4 c q+t s\right) d+2 c q-2 c^{2} q-t s+t s c \\
b_{2}=\left(2+q^{2}\right) d^{2}+\left(-t s q-2 c q^{2}-s^{2}-4 c\right) d+2 c^{2}+c^{2} q^{2}+s^{2}-t^{2} c-2+t^{2}-t s c q \\
b_{3}=-2 d^{2} q+(t s+2 q+4 c q) d+t^{2} c q-2 c q-2 c^{2} q-t s+t s c \\
b_{4}=1-t^{2} c-2 d+2 c+c^{2}-2 c d+d^{2}
\end{array}\right.
$$

Compute the subresultant sequence[13] for $f_{2}$ and $f_{4}$ with respect to $x$. Let the final two polynomials in the sequence be $f_{5}$ and $f_{6}$ respectively. We have

$$
f_{5}=i_{5} x-u_{5}, \quad f_{6}=i_{6} a^{8}-u_{6}
$$

where $i_{5}, u_{5}, i_{6}, u_{6}$ are polynomials in the parameters $\mathcal{U}$ and $u_{6}$ is of degree less than eight in $a$; These polynomials can be found at http://www.mmrc.iss.ac.cn/ ${ }^{\sim}$ xgao/paper/gaopnpa.pdf. Substituting $x$ by $u_{5} / i_{5}$ in $f_{1}$ and $f_{3}$ and clear denominators, we have

$$
f_{7}=i_{7} y-u_{7}, \quad f_{8}=i_{8} z-u_{8}
$$

where $u_{7}=(1-a+b) u_{5}^{2}+(q a-b q) u_{5} i_{5}+i_{5}^{2}(-a+b-1), i_{7}=r u_{5} i_{5}-p i_{5}^{2}, u_{8}=$ $(d-c+1) u_{5}^{2}+(c q-d q) u_{5} i_{5}+(-c+d-1) i_{5}^{2}, i_{8}=s u_{5} i_{5}-t i_{5}^{2}$. From $f_{5}=f_{7}=f_{8}=0$, we may represent $x, y$ and $z$ as rational functions in the parameters $\mathcal{U}$.

$$
x=\frac{u_{5}}{i_{5}}, y=\frac{u_{7}}{i_{7}}, z=\frac{u_{8}}{i_{8}} .
$$

Substituting $x, y, z$ by $\frac{u_{5}}{i_{5}}, \frac{u_{7}}{i_{7}}, \frac{u_{8}}{i_{8}}$ in $h_{5}$ and clear the denominators, we have

$$
f_{9}=i_{9} e-u_{9}
$$

where $u_{9}=-i_{5}^{2} i_{3}^{2} u_{2}^{2}-i_{5}^{2} i_{2}^{2} u_{3}^{2}+u i_{5}^{2} i_{2} i_{3} u_{2} u_{3}$ and $i_{9}=-i_{2}^{2} i_{3}^{2} u_{5}^{2}-i_{5}^{2} i_{2}^{2} i_{3}^{2}+q i_{5} i_{2}^{2} i_{3}^{2} u_{5}$. 
Lemma 3.5 For a set of parametric values, if $f_{6}=0, f_{9}=0, i_{5} i_{6} i_{7} i_{8} i_{9} \neq 0$, then equation system (2) has one set of solutions which can be computed with (8) and $w=x^{2}+1-x q$.

The proof will be given in the Appendix.

The following is a sketch of the proof for Theorem 3.4. From the computation process, the physical parameters must satisfy two equations $f_{6}=0, f_{9}=0$. There are eleven parameters in $\mathcal{U}$ and equations $f_{6}=0, f_{9}=0$ will reduce the number of free parameters by two. Intuitively speaking, the set of physical parameters $\mathbf{P}$ should be of dimension nine. Let $\mathbf{I}=\operatorname{Zero}\left(i_{5} i_{6} i_{7} i_{8} i_{9}\right)$. For a $\mathcal{U}_{0} \in \mathbf{P}-\mathbf{I}, x, y, z$ can be solved with (8). We need only to show that the dimension of $\mathbf{P} \cap \mathbf{I}$ is less than that of $\mathbf{P}$.

\section{$3.2 \quad$ Experimental Result}

We give the following procedure to solve the $\mathrm{P} 4 \mathrm{P}$ problem. By Lemma 3.5, if the parameters are from real observations, that is, the $\mathrm{P} 4 \mathrm{P}$ problem has at least one solution for these parameters, then the procedure will solve the $\mathrm{P} 4 \mathrm{P}$ problem with probability one.

- Compute the $l_{1}, l_{2}, l_{3}, l_{4}, l_{5}, l_{6}$ and $p, q, r, s, t, u$ from the control points, the image points and the camera calibration matrix.

- Compute the $a, b, c, d, e$ as follows. $a=\frac{l_{1}}{l_{3}}, b=\frac{l_{2}}{l_{3}}, c=\frac{l_{4}}{l_{3}}, d=\frac{l_{5}}{l_{3}}, e=\frac{l_{6}}{l_{3}}$.

- Compute $(x, y, z, w)$ as follows. $x=\frac{u_{5}}{i_{5}}, y=\frac{u_{7}}{i_{7}}, z=\frac{u_{8}}{i_{8}}, w=\frac{u_{5}^{2}+i_{5}^{2}-q u_{5} i_{5}}{i_{5}^{2}}$ where $i_{5}, u_{5}, i_{7}, i_{8}, u_{7}, u_{8}$ may be computed with the formulas given after equations (6) and (7). If $i_{5} i_{7} i_{8}=0$, the computation fails.

- Compute $X, Y, L, Z$ as follows: $L=\sqrt{l_{3} / w}, X=x L, Y=y L, Z=z L$. By Lemma 3.5, $X, Y, L, Z$ are a set of solutions to the $\mathrm{P} 4 \mathrm{P}$ problem.

We will discuss the accuracy and the stability of the algorithm. The following experiments are done with Maple.

The optical center is located at the origin and the matrix of camera's intrinsic parameters is assumed to be the identity matrix. At each trial, four non-coplanar controls points are generated at random within a cube centered at $(0,0,50)$ and of dimension $60 \times 60 \times 60$.

In the first experiment, we test the accuracy of our algorithm with a large set of data. For a set of solutions obtained with the algorithm, we substitute them into (2) and check whether the substituted values are zero or not. We take 1000 sets of parameters randomly. The maximal substituted value into $(2)$ is $0.1 * 10^{-9}$, which is satisfactory. 
In the second experiment, we test the stability of our algorithm with a larger set of data. One hundred trials are carried out and one hundred sets of parameters are computed for each trial. For each set of parametric values, two sets of solutions are computed: one with the original control points denoted by $S=(X, Y, L, Z)$; the other with the control points perturbed by random noises denoted by $\hat{S}=(\hat{X}, \hat{Y}, \hat{L}, \hat{Z})$. Similar to [10], if $\max (|X-\hat{X}|,|Y-\hat{Y}|,|L-\hat{L}|,|Z-\hat{Z}|)<1.5$, then $S$ and $\hat{S}$ are considered as the same solution. In trial $i$, let $n_{i}$ be the number of the parametric values such that the two results are the same. Let $\tilde{n}=\left(\sum_{i=1}^{100} n_{i}\right) / 100, \frac{\|100-\tilde{n}\|}{100}$ the absolute error, and $\frac{\sum_{i=1}^{100} \widetilde{X}_{i}}{100}$ the relative error where $\tilde{X}_{i}$ is the maximal value of $\max \left(\frac{|X-\hat{X}|}{|X|}, \frac{|Y-\hat{Y}|}{|Y|}, \frac{|L-\hat{L}|}{|L|}, \frac{|Z-\hat{Z}|}{|Z|}\right)$ for the one hundred sets of parametric values in the $i$-th trial. The experimental results are listed in the following table, from which we can observe that the algorithm yields very graceful degradation with increasing noises and is, therefore very stable.

\begin{tabular}{|c|c|c|c|c|c|c|c|c|}
\hline Noise Level & 0.001 & 0.01 & 0.1 & 0.5 & 1.0 & 2.0 & 3.0 & 4.0 \\
\hline Absolute error & 0 & 0 & 0 & 0 & 0.09 & 0.55 & 0.81 & 0.91 \\
\hline Relative error & $1.2 * 10^{-5}$ & $1.2 * 10^{-4}$ & 0.0012 & 0.0061 & 0.012 & 0.025 & 0.035 & 0.046 \\
\hline
\end{tabular}

Table 2. Error for the Algorithm

\section{Conclusion}

For the P4P problem, we proved that the probability for them to have a unique solution is one. We further show that the unique solution to the $\mathrm{P} 4 \mathrm{P}$ problem could be represented by a set of rational functions in the parameters and to solve these problems with the rational functions is quite stable and accurate.

Theoretically, we may obtain the explicit conditions for the P4P problem to have one, two, ... solutions. But, these conditions will be quite complicated and are computationally very difficult to get. On the other hand, the probabilities obtained in this paper give clear information on the distribution of the solutions for the P4P problems and may be used as a guidance on the number of solutions during the solving process.

\section{Appendix: Proof for Theorem 3.4}

We need several concepts from algebraic geometry which may be found in $[8,13,19]$.

Proof for Lemma 3.5. Continue with the computation in Section 3.2. Note that $f_{6}$ in (6) is free of $e$, then the following is an irreducible ascending chain $[13,19]$ under the 
variable order $b<c<d<p<q<r<s<t<u<a<e<x<y<z<w$.

$$
\left\{\begin{array}{l}
f_{6}=i_{6} a^{8}-u_{6}, f_{9}=i_{9} e-u_{9} \\
f_{5}=i_{5} x-u_{5}, f_{7}=i_{7} y-u_{7}, f_{8}=i_{8} z-u_{8}, q_{2}=-w+x^{2}+1-x q
\end{array}\right.
$$

We may check that the pseudo-remainders of the polynomials in (2) with respect to (10) are zero. Hence, we have the following remainder formulas (see [19, 13] for definition)

$$
J^{s_{i}} q_{i}=b_{i, 1} f_{6}+b_{i, 2} f_{9}+b_{i, 3} f_{5}+b_{i, 4} f_{7}+b_{i, 5} f_{8}+b_{i, 6} q_{2}, i=1, \ldots, 6
$$

where $J=i_{5} i_{6} i_{7} i_{8} i_{9}, s_{i}, i=1, \ldots, 6$ are non-negative integers, and $b_{i, j}$ are polynomials. From equations (11), we have

$$
\operatorname{Zero}((2))=\operatorname{Zero}((10) / J) \cup \operatorname{Zero}((2) \cup\{J\})
$$

Let $S_{0}=\operatorname{Zero}\left(\left\{f_{6}, f_{9}\right\} / J\right)$. Then $S_{0}$ is a quasi-variety for parameters $\mathcal{U}$ with dimension nine. For a set of values $\eta \in S_{0}$, since $J(\eta) \neq 0$, we may solve $x, y, z$, w with $f_{5}=f_{7}=$ $f_{8}=q_{2}=0$ in (10). Let the solutions be $x_{0}, y_{0}, z_{0}, w_{0}$. Substitute $\eta, x_{0}, y_{0}, z_{0}, w_{0}$ into (11). Since $J(\eta) \neq 0$, we have $q_{i}\left(\eta, x_{0}, y_{0}, z_{0}, w_{0}\right)=0, i=1, \ldots, 6$. Then $\eta, x_{0}, y_{0}, z_{0}, w_{0}$ consist of a set of solutions to (2). By the computation procedure, the polynomials in (10) are linear combinations of $q_{i}$. Then this is the only solution to (2).

Proof for Theorem 3.4. Use the notations introduced in the proof of Lemma 3.5. Since $w=x^{2}+1-x q>0$, we have $\operatorname{Zero}((2))=\operatorname{Zero}((2) / w)$. Under the variable order $x<y<z<p<q<r<u<t<w<a<b<c<d<e$, (2) is an irreducible ascending chain $[19,13]$. The initial of the polynomials in $(2)$ is $w$. Then Zero $((2) / w)$ is an irreducible quasi variety whose dimension is the number of the variables minus the number of polynomials in $(2)$, which is $15-6=9$. Since the pseudo-remainder of $J$ with respect to $(2)$ is not zero, $J$ does not vanish on $\operatorname{Zero}((2) / w)$. By the affine dimension theorem [8], $Z_{0}=\operatorname{Zero}((2) \cup\{J\} / w)$ is of lower dimension than nine. Applying Wu-Ritt Zero decomposition $[13,19]$, we have

$$
\operatorname{Zero}((2) \cup\{J\})=\cup_{j=1}^{d} \operatorname{Zero}\left(C_{i} / J_{j}\right)
$$

where $C_{j}$ are irreducible ascending chains $[13,19]$ and $J_{j}$ are the products of initials of the polynomials in $C_{j}$. Since $Z_{0}$ is of dimension less that nine, we have $\left|C_{j}\right|>6$. Let $A_{j}=C_{j} \cap Q[\mathcal{U}]$. By Lemma 2.1, the $\mathrm{P} 4 \mathrm{P}$ problem has a finite number of solutions. Then each $C_{j}$ contains four polynomials with leading variables $x, y, z, w$. As a consequence, $\left|A_{j}\right|>2$. We may assume that $J_{j}$ are polynomials free of $x, y, z, w[19]$. Let $S_{j}=$ $\operatorname{Zero}\left(A_{j} / J_{j}\right), j=1, \ldots, d$. Since $\left|A_{j}\right|>2, S_{j}$ is of dimension less than nine. Then $\mathbf{S}$ is the set of the parametric values for which the $x, y, z, w$ have complex solutions. $S_{0}$ is of dimension nine and $S_{j}, j>0$ are of dimension lower than nine. 
The physical parametric set could be obtained as follows

$$
\mathbf{P}=\cup_{j=0}^{d} S_{i}^{\prime}
$$

where $\mathbf{S}_{j}^{\prime}$ is a semi-algebraic set obtained by adding some inequalities to $\mathbf{S}_{i}$ so that $x, y, z, w$ have positive solutions [5]. Let

$$
\mathbf{T}=\cup_{j=1}^{d} S_{i}^{\prime}
$$

Since $\mathbf{S}_{i}^{\prime} \subset \mathbf{S}_{i}$, the dimension of $\mathbf{T}$ is less than nine. By Lemma 3.5, for parametric values in $\mathbf{P}-\mathbf{T}$, we may solve $x, y, z$ with (8). We now need only to show that the dimension of $\mathbf{P}$ is nine. Let $\mathbf{N}$ be the semi-algebraic set of dimension nine defined in $\mathbb{R}^{9}$ by $x>0, y>0, z>0,|p|<2,|q|<2,|r|<2,|s|<2,|t|<2,|u|<2$. We know that $w=x^{2}+1-x q>0$. Since $|r|<2, a=\frac{(x-y)^{2}+(2-r) x y}{w}>0$ for values from N. Similarly, $b, c, d, e$ are also positive. Then we may define a rational map from $\mathbf{N}$ to $\mathbf{P}$ as follows:

$$
\begin{aligned}
& p=p, q=q, r=r, s=s, t=t, u=u \\
& a=\frac{x^{2}+y^{2}-x y r}{x^{2}+1-x q}, b=\frac{y^{2}+1-y p}{x^{2}+1-x q}, c=\frac{x^{2}+z^{2}-x z s}{x^{2}+1-x q}, d=\frac{z^{2}+1-z t}{x^{2}+1-x q}, e=\frac{y^{2}+z^{2}-y z u}{x^{2}+1-x q}
\end{aligned}
$$

We may also define a rational map from $\mathbf{P}$ to $\mathbf{N}$ as follows.

$$
p=p, q=q, r=r, s=s, t=t, u=u, x=\frac{u_{5}}{i_{5}}, y=\frac{u_{7}}{i_{7}}, z=\frac{u_{8}}{i_{8}}
$$

For $J \neq 0$, map (14) has meanings. We may prove that (13) and (14) are inversion maps and thus are biratiobal maps between $\mathbf{N}$ and $\mathbf{P}$. Therefore, $\mathbf{P}$ is of dimension nine.

\section{References}

[1] M.A. Abidi and T. Chandra, A New Efficient and Direct Solution for Pose Estimation Using Quadrangular Targets: Algorithm and Evaluation, IEEE Tran. on PAMI, 17(5), 534-538, 1995.

[2] O. Faugeras, Three-Dimensional Computer Vision, a Geometric Viewpoint, MIT Press, 1993.

[3] M.A. Fishler and R.C. Bolles, Random Sample Consensus: A Paradigm for Model Fitting with Applications to Image Analysis and Automated Cartomated Cartography, Communications of the ACM, 24(6), 381-395, 1981.

[4] S. Ganapathy, Decomposition of Transformation Matrices for Robot Vision, Proc. IEEE Conf. Robotics abd Automation, 130-139, IEEE Press, 1984.

[5] X.S. Gao, X.R. Hou, J.L. Tang and H. Cheng, Complete Solution Classification for the Perspective-Three-Point Problem,IEEE Tran. PAMI, 25(8), 930-943, 2003. 
[6] R.M. Haralick, C. Lee, K. Ottenberg and M. Nolle, Analysis and Solutions of the Three Point Perspective Pose Estimation Problem, Proc. of the Int. Conf. on Computer Vision and Pattern Recognition, 592-598, 1991.

[7] R. Hartley and A. Zisserman, Multiple View Geometry In Computer Vision, Cambridge University Press, 2000.

[8] R. Hartshorne, Algebraic Geometry, Springer-Verlag, Berlin, 1977.

[9] R. Horaud, B. Conio and O. Leboulleux, An Analytic Solution for the Perspective 4-Point Problem, CVGIP, 47, 33-44, 1989.

[10] Z.Y. Hu and F.C. Wu, A Note on the Number Solution of the Non-coplanar P4P Problem, IEEE Tran. on PAMI, 24(4), 550-555, 2002.

[11] Z.Y. Hu and F.C. Wu, A Study on the P5P Problem, Chinese Journal of software, 12(5), 768-775, (in Chinese), 2001.

[12] Y. Hung, P. Yeh, and D. Harwood, Passive Ranging to Known Planar Points Sets, Proc. IEEE Int. Conf. on Rob. and Auto., 1,80-85, St. Louis, 1985.

[13] B. Mishra, Algorithmic Algebra, Springer-Verlag, New York, 1993.

[14] S. Maybank, Theory of Reconstruction from Image Motion, Springer-Verlag New York, 1992.

[15] K. Killian, Ueber das Rueckwaertseinschneiden im Raum, Oesterreichische Zeitschrift fuer Vermessungswcse, 4, 97-104, and 5, 171-179, 1955.

[16] K. Killian and P. Meissl, Zue Loesung Geometrisch Ueberbestimmter Probleme, Oesterreichische Zeitschrift fuer Vermessungswcse, 64(3/4), 81-86, 1977.

[17] L. Quan and Z. Lan, Linear N-Point Camera Pose Determination, IEEE Tran. on PAMI, 21(8), 774-780, 1999.

[18] B.P. Wrobel, Minimum Solutions for Orientation, in Proc. Workshop Calibration and Orientation of Cameras in CV, Washington D.C., Springer, August, 1992.

[19] W.T. Wu, Mathematics Mechanization, Science Press/Kluwer Aacademic Publishers, Beijing, 2000.

[20] W.J. Wolfe, D. Mathis, C. Weber and M. Magee, The Perspective View of Three Points, IEEE Tran. on PAMI, 13(1), 66-73, 1991.

[21] J.S.C. Yuan, A General Photogrammetric Method for Determining Object Position and Orientation, IEEE Tran. on Robotics and Automation, 5(2), 129-142, 1989. 